\title{
Ruler-Based Automatic Stitching of Spatially Overlapping Radiographs
}

\author{
André Gooßen ${ }^{1}$, Mathias Schlüter ${ }^{2}$, Marc Hensel$^{2}$, Thomas Pralow ${ }^{2}$, \\ Rolf-Rainer Grigat ${ }^{1}$ \\ ${ }^{1}$ Vision Systems, Hamburg University of Technology, D-21079 Hamburg \\ ${ }^{2}$ General X-Ray, Philips Medical Systems, D-22335 Hamburg \\ andre.goossen@tu-harburg.de
}

\begin{abstract}
We present an algorithm for fast automatic registration of spatially overlapping radiographs. It possesses strong robustness against noise, feature masking and feature displacement. Pivotal for this algorithm is an actual interpretation of the stitching feature instead of a simple detection. The proposed method has been evaluated on 3000 clinical radiographs and proved to be a powerful enhancement of established automatic registration algorithms.
\end{abstract}

\section{Introduction}

When imaging long parts of the human body, e.g. legs or spine, in conventional screen-film technique, special cassettes and films of extended length are utilized. Migration to digital radiography limits the image size due to the sensitive area of flat-panel detectors. In order to reproduce the behavior of conventional radiography a large image is assembled from multiple exposures with a small spatial overlap. As the detector has to be moved to the next position between subsequent exposures there is a risk that patient movement or breathing might produce inconsistent content. Refer to Figure 1 for a depiction of the acquisition.

Known algorithms for automatic registration either apply feature-based registration [1], landmark-based registration [2] or plain image-similarity measures [3]. Although proven in computed radiography (CR), feature-based algorithms are not applicable for digital radiography (DR) as the patient may have moved against the feature in subsequent exposures and hence matching the feature is not sufficient in DR. Image-similarity has to deal with ambiguities of the similarity measurement, e.g. recurring structure of the spine, within the region of possible solutions and thus requires a qualified a priori estimation within the large possible overlap spanned by DR images. Landmark-based registration performs well for known content, but since modeling the content of arbitrary medical images is considered extremely difficult or impossible [4], it fails for digital radiographs.

The proposed method overcomes these problems by combining feature-based registration and similarity measurement. However instead of computing interimage correspondences our method derives correspondences between image coordinates and real world coordinates by not only detecting but also interpreting the feature, metering its information. 
Fig. 1. Multiple radiograph acquisition. The patient stands in front of the flat-panel detector. While the detector moves up and down to reach the different exposure positions, the X-ray tube is panned around its rotational axis to expose the detector. An X-ray ruler brought between patient and detector serves as the feature for later composition and distortion removal.

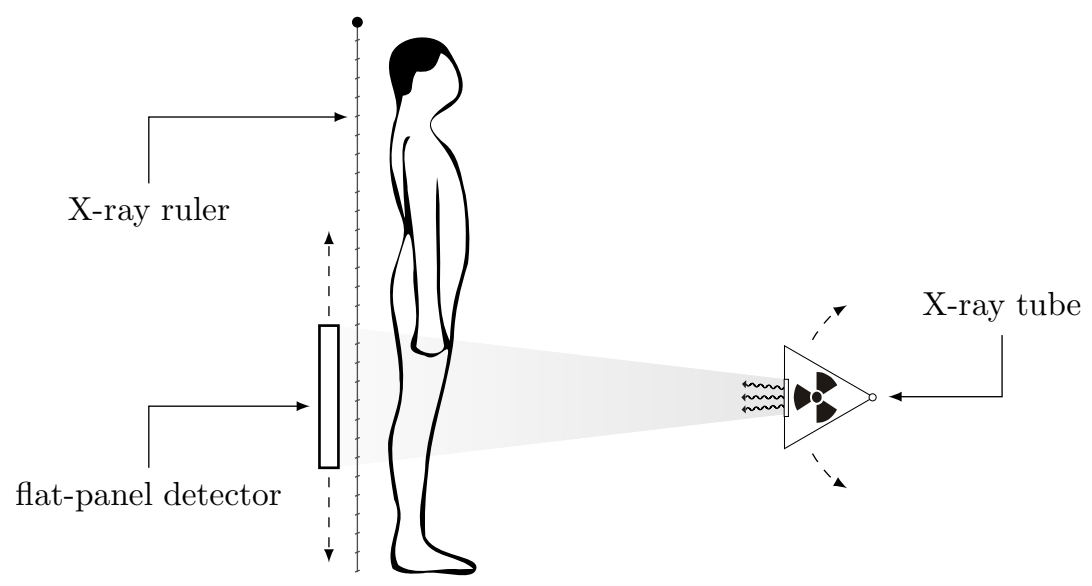

\section{Methods and Materials}

The proposed method consists of two steps complementing one another. The ruler recognition algorithm locates the feature, i.e. the X-ray ruler and interprets markers and digits on this feature to extract the global meaning. The automatic stitching computes an estimated translation for subsequent images featuring the ruler recognition and refines the translation to match anatomy.

\subsection{Ruler Recognition}

The algorithm consists of four basic stages executed in a sequential manner:

1. Localization of the ruler line,

2. Binarization of the ruler part,

3. Marker and digit localization,

4. Digit recognition and interpretation.

The first stage utilizes a discrete Radon transform on the source image to locate and parameterize the ruler line. With the line angle and distance to the origin known, the ruler sub-part is deskewed and cropped out of the source image. Finally the crop is binarized using a local-adaptive technique with a threshold that varies along a sliding window over the ruler height. This introduces invariance against translation and rotation of the ruler as well as against spatially varying image intensity or contrast. The resulting image forms the input for subsequent processing stages. 
With the ruler line detected, a profile along the line is generated by projection onto the vertical axis. Due to the recurring scale markers and numbers, this profile shows periodicity and hence the marker period and phase can be determined by autocorrelating the profile. Detected marker positions have to be corrected in a hit-or-miss transformation and markers missing due to coverages or bad signalto-noise ratio are interpolated by linear regression. This interpolation allows the assembling of images with no visible feature within the overlapping region.

To detect numbers at the refined marker locations, a search for connected pixels is performed in each row containing a marker. The resulting groups of connected pixels serve as candidates for digit recognition. The bounding box size of these candidates forms a criterion for filtering out noise (e.g. very small boxes) and artifacts (e.g. very large boxes, wrong aspect ratios, etc.) and for picking the selection to perform the optical character recognition on.

The final stage performs optical character recognition using a dedicated template matching algorithm [5]. In order to correct missing digits or numbers and false recognitions, a virtual ruler is moved along the detected digits. Matching digits increase the score for a certain position, mismatched digits degrade it. Due to the high redundancy, the displacement of maximum congruency determines the positioning of the ruler, i.e. the ruler "snaps in" at the correct position yielding a set of global correspondences of image coordinates to real-world coordinates.

Figure 2 depicts the detected ruler geometry for a selection of clinical images to point out the capabilities of the proposed method. The algorithm is invariant against ruler type and scale font as well as ruler translations, rotations and scaling. Missing geometry is extrapolated automatically to restore information in covered overlap areas.

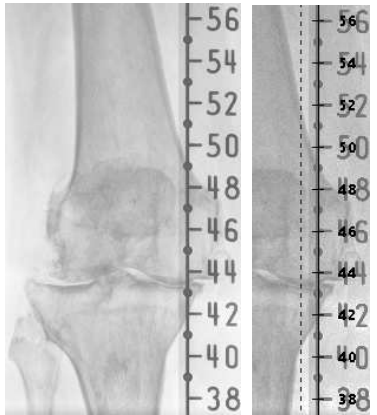

(a) original and overlay

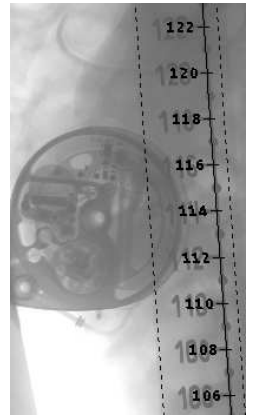

(b) tilted ruler

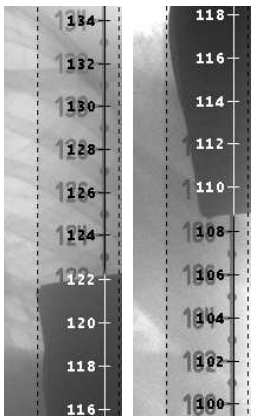

(c) partial coverage

Fig. 2. Detected ruler geometry within four crops of clinical radiographs. The results of the recognition have been overlaid onto the original radiograph. (a) Comparison of original radiograph and detected ruler geometry within an examination of a leg. (b) Detected geometry for a tilted ruler. (c) Detected (black) and extrapolated (white) geometry of a partially covered ruler within a pair of thoracic images. 


\subsection{Automatic Stitching}

Repeating the ruler recognition algorithm for two subsequent images and taking their correspondences yields an initial estimation for the translation between these images. Since the estimation maps ruler coordinates of both images onto each other, the translations have to be refined to map corresponding parts of the anatomy. This is done by performing a correlation of the images in a small window of half the ruler period around the calculated estimation, avoiding ambiguities of the similarity measure. In order to introduce invariance against local intensity, a gradient correlation $[6,7]$ is used to match image content. For this purpose we project the anatomy fraction and differentiate the derived profile. To deal with noise a low pass filter smooths the profile. Maximizing the normalized cross correlation yields the final vertical and horizontal offset.

\subsection{Experiments}

An image pool of 3000 radiographs has been used to evaluate the proposed method. The image pool has been acquired on two clinical sites and contains all kinds of anatomies and geometries in two or three single exposures, i.e. one or two stitches per images.

As there is no true optimum for the translation between two subsequent images, the major difficulty for evaluation is the creation of a valid reference. For the evaluation of our method, reference translations have been acquired from manual stitching of the test images by 6 observers. To eliminate influences due to inter-observer variability in manual stitching, we performed a study on a representative subset of 42 image pairs. Each observer had to perform stitching on all of these images and the deviation between stitching translations for the same image derived by different observers was measured in Euclidean distance. The distribution of deviations (cp. histogram in Table 1) determined the failure threshold for automatic stitching to $3 \mathrm{~mm}$.

Images where the deviation between automatically computed translation and manual reference exceeded the threshold are therefore considered as failures.

\section{Results}

Experiments have shown that the proposed algorithm locates the correct translation in respect to the manual reference within a very small window around the desired reference for the majority of tests. 67 radiographs have been rejected by the algorithm due to operational errors of the acquisition staff, e.g. missing rulers or wrong apertures. This results in 1814 pairs of subsequent images forming the evaluation set.

1611 out of these 1814 image pairs containing valid rulers are registered with a deviation of less than $1 \mathrm{~mm}$. The translations for $99.3 \%$ of all valid image pairs lie in the range of less than $3 \mathrm{~mm}$ devation. Thus the failure rate is $0.7 \%$ for the proposed algorithm. Table 1 lists the results of the evaluation for the proposed method. The average processing time is $158 \mathrm{~ms}$ per computed translation for an image pair on an Intel Pentium D $2.8 \mathrm{GHz}$. 
Table 1. Results of automatic stitching for 1814 valid image pairs. $\Delta$ represents the measured Euclidean distance of the calculated translation to the reference translation. Deviations $>3 \mathrm{~mm}$ sum up to a failure rate of $0.7 \%$. The histogram contrasts the results of the inter-observer study and the proposed method.

\begin{tabular}{ccc}
\hline$\Delta$ & occurrence & fraction \\
\hline $0-1 \mathrm{~mm}$ & 1611 & $88.8 \%$ \\
$1-2 \mathrm{~mm}$ & 147 & $8.1 \%$ \\
$2-3 \mathrm{~mm}$ & 43 & $2.4 \%$ \\
$3-4 \mathrm{~mm}$ & 7 & $0.4 \%$ \\
$4-5 \mathrm{~mm}$ & 4 & $0.2 \%$ \\
$>5 \mathrm{~mm}$ & 2 & $0.1 \%$ \\
\hline
\end{tabular}

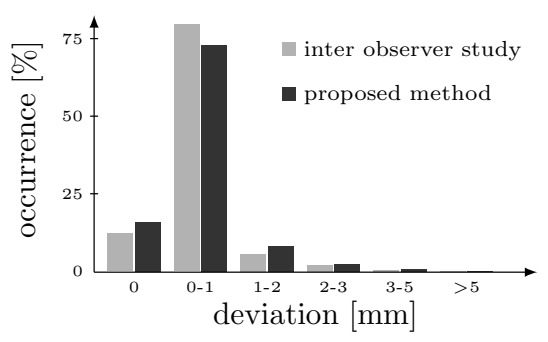

\section{Discussion}

The proposed method decreases the failure rate of automatic stitches to $0.7 \%$. As is apparent from the results, the performance of the proposed method comes close to the lower boundary formed by the accuracy of manual stitching references. The low processing time results in minimum costs for ruler recognition within the image processing chain.

The high robustness has been proved by processing a large number of clinical images. Manual interaction is strongly reduced by the proposed algorithm and the risk of false automatic stitches, possibly resulting in unnecessary treatment, is minimized.

\section{References}

1. Čapek M, Wegenkittl R, Felkel P. A fully automatic stitching of 2D medical data sets. BIOSIGNAL. 2002;16:326-8.

2. Johnson HJ, Christensen GE, Little JA, et al. Consistent landmark and intensitybased image registration. IEEE Trans Med Imaging. 2002;21(5):450-61.

3. Gramer M, Bohlken W, Lundt B, et al. An algorithm for automatic stitching of CR X-ray images. Advances in Medical Engineering. 2007;114:193-8.

4. Lehmann T. Medizinische Bildverarbeitung. In: Kramme R, editor. Medizintechnik: Verfahren, Systeme, Informationsverarbeitung. 2nd ed. Springer; 1997. p. 588-612.

5. Harmon LD. Automatic recognition of print and script. Proc IEEE. 1972;60(10):1165-76.

6. Heipke C. Overview of image matching techniques. OEEPE Official Publications. 1996;33:173-89.

7. Zitová B, Flusser J. Image registration methods: A survey. Image Vis Comput. 2003;21(11):977-1000. 